La

Révolution

française

\section{La Révolution française}

Cahiers de l'Institut d'histoire de la Révolution française

$16 \mid 2019$

1801-1840 - Haïti, entre Indépendance et

Restauration

\title{
Guerre, commerce et migrations dans le premier empire colonial français : approche micro- historique
}

Pierre Force

\section{OpenEdition}

\section{Journals}

Édition électronique

URL : http://journals.openedition.org/lrf/2723

DOI : $10.4000 /$ Irf. 2723

ISSN : 2105-2557

Éditeur

IHMC - Institut d'histoire moderne et contemporaine (UMR 8066)

Référence électronique

Pierre Force, «Guerre, commerce et migrations dans le premier empire colonial français : approche micro-historique », La Révolution française [En ligne], 16 | 2019, mis en ligne le 20 juin 2019, consulté le 22 juin 2019. URL : http://journals.openedition.org/lrf/2723 ; DOI : 10.4000//rf.2723

Ce document a été généré automatiquement le 22 juin 2019

(c) La Révolution française 


\title{
Guerre, commerce et migrations dans le premier empire colonial français : approche micro- historique
}

\author{
Pierre Force
}

1 Je propose ici quelques réflexions sur la méthode et les conclusions de mon livre paru en 2016, Wealth and Disaster'. J'insisterai en particulier sur les points de contact avec l'ouvrage de Paul Cheney, Cul de Sac ${ }^{2}$, publié presque simultanément. Je dirai aussi quelques mots du débat entre Paul Cheney et les auteurs de The Plantation Machine, Trevor Burnard et John Garrigus, sur les rapports entre capitalisme et esclavage dans l'économie de plantation.

\section{Poser des questions en micro-histoire}

2 Si l'on m'avait dit, il y a dix ans, que j'écrirais un livre sur les flux migratoires entre un village pyrénéen et Saint-Domingue, j'aurais trouvé l'idée saugrenue. L'idée de ce livre ne m'est venue ni de mes lectures, ni de mes recherches précédentes. Comme c'est souvent le cas en micro-histoire, c'est une trouvaille archivistique qui est à l'origine du projet.

3 En novembre 2008, alors que j'étais en congé sabbatique en France, la commune de La Bastide Clairence, dans les Pyrénées-Atlantiques, fit l'acquisition d'une maison à laquelle était rattaché un trinquet (salle couverte destinée à la pratique de la pelote basque). Ce lieu était pour moi très familier, car la maison avait appartenu à mes grands-parents maternels et j'avais moi-même, enfant et adolescent dans les années 60 et 70 , maintes fois joué à la pelote basque dans ce trinquet pendant les vacances d'été. Le trinquet semblait très ancien, et certains prétendaient qu'il s'agissait d'un jeu de paume reconverti pour la pratique de la pelote basque. La municipalité souhaitait faire classer le bâtiment. On avait fait venir des experts du ministère de la culture qui s'étaient montrés sceptiques. Je proposai au maire de La Bastide Clairence, Léopold Darritchon, d'aller consulter les 
archives du cadastre et les archives notariales pour découvrir les anciens propriétaires du lieu et recueillir des preuves de l'ancienneté du bâtiment. Je pensais que cette recherche prendrait une après-midi ou deux. Elle a duré plusieurs années.

4 Je découvris d'abord que le trinquet avait été acquis en 1899 dans une vente aux enchères par mon arrière-grand-père, Bernardin Hapette, charpentier à La Bastide Clairence. Il s'agissait en effet d'une salle de jeu de paume que mon arrière-grand-père réaménagea pour la pratique de la pelote basque quelques années après. Fait remarquable, les vendeurs de 1899, qui possédaient la salle de jeu de paume en indivision, étaient tous membres d'une même famille établie en "République orientale de l'Uruguay (deux cordonniers à San José, un militaire à Montevideo et une veuve sans profession à Montevideo). Ce fait piqua ma curiosité mais ne m'étonna qu'à moitié, l'émigration basque et béarnaise vers l'Argentine, l'Uruguay et le Chili au $19^{\mathrm{e}}$ siècle étant un phénomène bien connu. Remontant dans le temps, je pus établir que le jeu de paume avait été acquis en 1823 par le grand-père des cordonniers uruguayens, Pierre Cornu, dit Cornu Galan, bonnetier à La Bastide Clairence. Le vendeur, Jacob Gomès, négociant à SaintEsprit-lès-Bayonne, était lui-même issu de la petite communauté juive de La Bastide Clairence: son grand-père, nommé lui aussi Jacob Gomès, avait été l'apothicaire de La Bastide Clairence au milieu du $18^{\mathrm{e}}$ siècle (une communauté juive sépharade existait à La Bastide Clairence depuis le début du $17^{\mathrm{e}}$ siècle, placée sous la protection du duc de Gramont). Jacob Gomès avait lui-même acquis le bâtiment cinq ans plus tôt, en 1818. En lisant l'acte de vente de 1818, je vis que les vendeurs, qui possédaient la salle de jeu de paume en indivision, était tous membres d'une même famille dispersée en plusieurs points de l'espace atlantique. Une sœur habitait Aïcirits, à vingt-cinq kilomètres de La Bastide Clairence. Un frère, résidant lui aussi à Aïcirits mais récemment arrivé d'Amérique, était domicilié à La Nouvelle Orléans. Le reste de la famille était domicilié à Cuba, principalement à Matanzas, mais aussi à La Havane.

5 La lecture de ce document me plongea dans la perplexité. Le nom de famille des propriétaires, Lamerenx, ne me disait rien. Aucun rapport de parenté ne semblait exister entre cette famille et les habitants actuels de La Bastide Clairence. Cependant, s'ils avaient été propriétaires du jeu de paume, il fallait bien qu'ils aient habité La Bastide Clairence à une époque antérieure. À ce stade, les membres de la famille Lamerenx n'étaient pour moi que des noms sur une page. Je ne savais d'eux que ce que je voyais sur un acte notarié de 1818, et les raisons de la dispersion de cette famille à travers l'espace atlantique m'étaient inconnues. La question qui guida mes premières recherches fut toute simple : «Bon sang, mais qui sont ces gens? ». Si l'émigration basque vers l'Amérique du Sud dans la seconde moitié du $19^{\mathrm{e}}$ siècle était un phénomène bien connu, il était beaucoup plus étonnant de découvrir des liens entre ce village pyrénéen et les Amériques au début du $19^{\text {e }}$ siècle. La Bastide Clairence n'est pas une ville côtière. C'est un bourg situé à vingtcinq kilomètres à l'intérieur des terres. L'idée que des habitants aient pu émigrer vers les Antilles ou la Louisiane au $18^{\mathrm{e}}$ siècle semblait surprenante. On n'en trouve en tout cas aucune trace dans la tradition orale ou la mémoire collective, alors que l'émigration plus récente vers l'Amérique du Sud est très présente dans les mémoires (de nombreuses familles de La Bastide Clairence ont des cousins plus ou moins lointains en Argentine).

6 Pour orienter mes recherches afin de résoudre cette énigme, je dus imaginer des hypothèses. Après avoir un peu lu et réfléchi, l'hypothèse qui m'apparut la plus probable fut la suivante : les propriétaires du jeu de paume descendaient d'un ancêtre commun qui était parti pour Saint-Domingue au $18^{\mathrm{e}}$ siècle, et la famille s'était réfugiée à Cuba suite à la 
Révolution haïtienne. Après quelques semaines de travail, cette hypothèse se révéla être la bonne. Je découvris ainsi assez rapidement qu'un membre de cette famille, qui apparaît dans les archives comme "le capitaine Lamerenx », avait été l'un des aides-de-camp de Toussaint Louverture en 1794.

Ces découvertes furent pour moi très signifiantes. Il y avait là un télescopage du familier et de l'étrange, du connu et du nouveau, qui attirait mon attention. Cette salle de jeu de paume était pour moi un lieu familier, provincial, évoquant l'ennui des après-midis d'été. Soudain, ce lieu que je croyais si bien connaitre apparaissait sous un jour entièrement nouveau, et lié à une figure de l'histoire universelle avec seulement un ou deux degrés de séparation. De surcroît, la présence du capitaine Lamerenx, propriétaire blanc, aux côtés du général noir précurseur de l'indépendance haïtienne, était un paradoxe demandant une explication. J'eus très tôt le sentiment que j'avais là le sujet d'un livre.

8 J'envisageai cependant d'autres sujets. En février 2011, la commune de La Bastide Clairence fit procéder sur mes conseils à une expertise dendro-chronologique de la charpente du jeu de paume qui donna l'année 1512 comme date probable de la construction du bâtiment. Mes propres recherches en archives me permirent d'identifier avec plus ou moins de précision les propriétaires du bâtiment, et ce jusqu'au début du $16^{\mathrm{e}}$ siècle. Il y avait là une aubaine pour la municipalité : le trinquet de La Bastide Clairence se révélait être le plus ancien jeu de paume du monde encore en activité (et cela probablement sans discontinuer depuis le début du $16^{\mathrm{e}}$ siècle). L'inscription du bâtiment à l'inventaire supplémentaire des monuments historiques fut obtenue sans difficulté quelques mois plus tard, et la salle de jeu de paume rénovée fut ouverte aux quelques amateurs de jeu de paume à l'ancienne ainsi qu'aux pratiquants plus nombreux de la pelote basque contemporaine.

9 M'étant documenté sur l'histoire architecturale des jeux de paume, je me rendis compte que la disposition intérieure du bâtiment était identique à celle qu'on aperçoit sur les croquis préparatoires que David avait effectués en vue de son célèbre tableau représentant le serment du jeu de paume. Là encore, un lieu familier et banal m'apparaissait sous un jour entièrement nouveau. Une expression anglaise, «hiding in plain sight » (littéralement, "se cacher en pleine vue »), rend assez bien la découverte de tout ce contexte historique. Le même objet, regardé un peu différemment, révèle des aspects insoupçonnés qui font entièrement réévaluer sa signification. J'envisageai d'écrire un livre qui serait l'histoire d'un lieu, ce jeu de paume construit au début du $16^{\mathrm{e}}$ siècle par de grands notables proches des souverains de Navarre. J'aurais reconstitué la vie des habitants successifs du lieu, allant jusqu'au $20^{e}$ siècle et aux soldats allemands que mes grands-parents furent forcés de loger pendant l'Occupation. Le manque d'archives pour les périodes les plus anciennes rendait le projet difficilement réalisable. Entretemps, j'avais découvert qu'au moins une demi-douzaine de familles de La Bastide Clairence avaient envoyé des émigrants à Saint-Domingue au $18^{\mathrm{e}}$ siècle (et j'avais aussi pris connaissance des travaux de Jacques de Cauna sur l'émigration des Aquitains aux Antilles au $18^{\mathrm{e}}$ siècle ${ }^{4}$ ). J'envisageai alors de placer mon récit à l'échelle du village et de raconter l'histoire des relations migratoires (allers et retours) entre La Bastide Clairence et l'espace caribéen aux $18^{\mathrm{e}}$ et $19^{\mathrm{e}}$ siècles. Cependant, un des aspects qui m'intéressaient le plus dans cette perspective micro-historique était l'étude détaillée de stratégies individuelles. Envisager la totalité des émigrants de ce village tout en accordant à chacun une attention détaillée aurait abouti à un récit trop fouillé et comportant trop de personnages. Je décidai de me limiter aux deux premiers émigrants et à leurs familles. J'ai 
déjà parlé du premier émigrant, Marc-Antoine Lamerenx, cadet d'une famille de toute petite noblesse, qui partit d'Aïcirits pour Saint-Domingue en 1729. Son neveu Jean-Pierre Lamerenx, natif de La Bastide Clairence, vint le rejoindre en 1764 à Saint-Martin-duDondon, dans les montagnes au sud du Cap-Français. Il y établit une plantation de café à proximité de celle que son oncle avait implantée dans les années 1740 , au commencement du boom du café. Le second émigrant dont je parle dans le livre est Jean Mouscardy, parti de La Bastide Clairence dans les années 1740, qui vint s'établir lui aussi à Saint-Martin-duDondon, et acheta ses premières terres en 1750 . Il bénéficia très probablement au départ du soutien de son compatriote Marc-Antoine Lamerenx, qui était son voisin au Dondon. Le récit de ces vies parallèles permet des comparaisons et des distinctions intéressantes entre ces deux personnes et ces deux familles à la fois très semblables et très différentes. Marc-Antoine Lamerenx et Jean Mouscardy étaient tous les deux de "grands blancs", planteurs de café et propriétaire chacun de plus de cent esclaves. Dans leur Basse-Navarre natale, l'un était noble et l'autre paysan. Ils avaient en commun le fait d'être des cadets. Lamerenx épousa une fille de planteurs. Ses enfants et ses neveux quittèrent tous SaintDomingue suite à la Révolution haïtienne. La plupart se réfugièrent à Cuba, où ils firent fortune à nouveau comme planteurs de café, reprenant très précisément la méthode qu'ils avaient suivie à Saint-Domingue (exploitation de terres acquises à bon marché avec réinvestissement immédiat des profits dans l'achat de nouveaux esclaves). Mouscardy avait, quant à lui, pour compagne sa «ménagère », Françoise Alzire, femme de couleur libre qui gérait sa maisonnée et avec qui il eut au moins six enfants. Mouscardy fut chassé de sa plantation quand ses esclaves se révoltèrent en 1791. Selon une légende familiale, en partie corroborée par ce qu'on peut inférer des archives, il fut protégé par ses enfants mulâtres et put se réfugier au Cap. Ruiné par l'incendie du Cap, dans lequel il avait perdu tout son argent et ses titres, il s'embarqua pour Boston en 1793 et parvint enfin à Bayonne, où il mourut en 1797. Ses enfants restèrent tous en Haïti. Son fils aîné, JeanThéodat Mouscardy, fut l'un des intendants de la maison du roi Christophe et finit sa carrière comme général dans l'armée haïtienne. Un de ses arrière-petits-enfants, Démesvar Delorme, joua un rôle notable dans l'histoire politique d'Haïti au $19^{\mathrm{e}}$ siècle, et est surtout connu comme l'un des pères fondateurs de la littérature haïtienne. Dans ses souvenirs d'enfance, Delorme parle de la tradition familiale selon laquelle l'un de ses ancêtres était un grand blanc nommé Mouscardy.

Je ne me suis donc pas lancé dans ces recherches pour confirmer ou infirmer une hypothèse de grande envergure. Je suis parti d'un problème limité. J'ai tâché de répondre à une question précise : comment se fait-il que deux cadets, Marc-Antoine Lamerenx et Jean Mouscardy, que les coutumes successorales de leur région destinaient à la pauvreté, soient devenus si riches? Cette question en appelait d'autres : qu'est-ce qui les a décidés à passer en Amérique? comment ont-ils fait fortune? combien ont-ils payé pour leur passage? combien leur ont coûté les terres et les esclaves? qui étaient leurs esclaves? quels étaient leurs rapports, y compris sexuels, avec leurs esclaves ? comment se sont-ils comportés avec les enfants qu'ils ont eus avec leurs esclaves? et que s'est-il passé quand leurs esclaves les ont mis dehors?

Un malentendu fréquent concernant la micro-histoire ou les études de cas consiste à se demander si le cas étudié est représentatif d'un phénomène plus vaste, et si l'on peut passer du particulier au général, ou du cas à la règle. En réalité, la règle est toujours déjà implicitement présente dans l'énoncé d'un cas. Ce qui fait d'un cas un cas, c'est précisément le fait qu'il semble contrevenir à la règle ${ }^{5}$. Ce qui fait de l'histoire de Marc- 
Antoine Lamerenx et de Jean Mouscardy des cas, c'est qu'ils soient devenus riches alors que les cadets étaient destinés à être pauvres. Le cas se présente comme une exception à la règle. Le travail de l'historien consiste à déterminer si l'exception confirme la règle ou si, au contraire, elle doit amener à la formulation d'une nouvelle règle. Autre exemple : une grande partie du chapitre 3 de mon livre est une discussion du cas de Jean-Pierre Lamerenx, neveu de Marc-Antoine, qui partit rejoindre son oncle au Dondon en 1764. Jean-Pierre était un aîné. Le fait qu'il ait décidé d'émigrer contrevient à la règle qui veut que ce soient les cadets qui partent. Ici encore, la question est de savoir si l'exception doit nous amener à une confirmation ou à une reformulation de la règle.

Pour reconstituer ainsi en détail les trajectoires individuelles de personnages obscurs, j'ai usé de moyens très modernes et d'autres beaucoup plus traditionnels. Le fait que mes personnages aient des patronymes inhabituels m'a permis de les identifier assez facilement lorsqu'ils apparaissaient dans telle ou telle base de données, et de les repérer dans tel ou tel endroit du monde où je n'aurais pas pensé à les chercher. J'ai bénéficié de la collaboration de généalogistes amateurs descendants de mes personnages, notamment Alina Arce, ingénieure à La Havane, et Christina Schutt, avocate à Port-au-Prince, toutes deux rencontrées sur des forums de généalogie sur Internet. Alina Arce, descendante des Lamerenx, a trouvé plusieurs documents essentiels concernant ces derniers aux archives nationales de Cuba. C'est Christina Schutt, descendante de Jean Mouscardy, qui m'a indiqué le passage des mémoires de Démesvar Delorme où il est question du général Mouscardy. Tout cet aspect de ma recherche aurait été impensable si je m'en étais tenu à des méthodes traditionnelles de consultation d'archives. Mes liens familiaux avec La Bastide Clairence m'ont donné accès à des documents qu'un autre historien n'aurait sans doute pas pu obtenir. Le maire de La Bastide Clairence m'a honoré de sa confiance en me donnant les clés de la mairie et du grenier où étaient déposées les archives municipales, qui n'étaient à l'époque ni cotées ni répertoriées (un groupe de bénévoles les a inventoriées depuis, de manière très compétente). C'est dans les archives municipales que j'ai trouvé, dans un cahier à moitié mangé par les rats, les dépositions de la sentence arbitrale de 1763 concernant le conflit dont je parle au chapitre 3 entre Mathieu Lamerenx et son épouse Anne de Marmont. Et c'est dans des archives privées, généreusement mises à ma disposition par Denis Dufourcq, que j'ai trouvé le détail des dépenses effectuées pour équiper Jean-Pierre Lamerenx avant son embarquement pour Saint-Domingue en 1764. C'est, je crois, le même mélange de chance et de persévérance qui a caractérisé les recherches de Paul Cheney. Ayant fait l'hypothèse qu'il existait sans doute une correspondance postérieure à 1789 dans les papiers de la famille La Ferronnays, il s'est adressé à la descendante d'une branche collatérale, et celle-ci a découvert cent soixante lettres inédites dans les archives familiales.

Comme beaucoup de travaux de micro-histoire, mon étude est, à certains égards, un travail d'anthropologie historique, et c'est à dessein que le premier chapitre s'ouvre sur une citation de la conférence de Lévi-Strauss intitulée "Histoire et ethnologie ». Une partie des discussions que je propose relève de ce que, en ethnologie, on appelle parfois " savoir indigène ». Les coutumes successorales pyrénéennes, l'exclusion des cadets, et le droit d'aînesse absolue m'étaient connus de manière anecdotique et par tradition familiale avant que je ne me plonge dans l'abondante littérature anthropologique et sociologique sur la question (au reste, j'ai été surpris de constater que ce qui, pour moi, n'était que des coutumes un peu bizarres du pays de mes grands-parents ait fait l'objet d'études si approfondies). En lisant les travaux de Pierre Bourdieu sur les stratégies 
matrimoniales des paysans du Béarn ${ }^{6}$, et ceux d'Henri Lefebvre sur les communautés paysannes pyrénéennes ${ }^{7}, \mathrm{j}^{\mathrm{j}}$ étais particulièrement conscient du fait qu'ils mobilisaient eux aussi un savoir indigène, ayant passé l'un et l'autre leur enfance et leur adolescence dans le Béarn rural. Cette posture a bien sûr ses dangers, mais je crois qu'elle offre aussi un point de vue incomparable sur une culture, qui est de pouvoir l'envisager à la fois de l'intérieur et de l'extérieur. C'est ce qui m'a permis, par exemple, de montrer quels étaient les enjeux économiques et symboliques de la dispute entre Mathieu Lamerenx et son épouse Anne de Marmont, derrière la technicité et la particularité du vocabulaire juridique des dépositions, qui auraient rebuté beaucoup d'autres chercheurs.

Dans l'introduction de son livre, Paul Cheney revendique l'héritage des travaux de Gabriel Debien. Je fais de même dans l'introduction du mien, où je rappelle que Debien a commencé à s'intéresser à l'histoire coloniale en fouillant dans les archives du HautPoitou (région située à une certaine distance de l'océan). Debien, pourrait-on dire, faisait de la micro-histoire avant la lettre. Dans un numéro des Annales de 1948, on trouve un compte-rendu de huit articles de Debien, tous publiés dans la Revue de la Société d'Histoire et de Géographie d'Haïti. L'auteur du compte-rendu y loue Debien d'avoir révélé « tout un complexe de problèmes, peu soupçonnés d'ordinaire ». Il ajoute que "l'explication va, sans méandres, du document original, classé, daté, vu de près, à la monographie à demiconstruite seulement, avec des amorces de systématisation, rien de plus $»^{8}$. Suit une note signée de Fernand Braudel, qui fait l'éloge des «richesses magnifiques des articles et études de G. Debien, ami des Annales, disciple de Marc Bloch, combattant efficace de ce qui est pour nous la bonne méthode, ouvrier d'une histoire digne de nous passionner ${ }^{9}$. »

Comme Debien, Paul Cheney et moi-même faisons usage de "papiers de famille", archives privées qui permettent d'éclairer des stratégies individuelles ou familiales beaucoup plus nettement que des documents déposés aux archives nationales ou départementales. C'est aussi dans ce type d'archives qu'on saisit le mieux la complexité des rapports entre l'espace colonial et l'espace métropolitain et qu'on voit à quel point il est nécessaire de les étudier ensemble. C'est en changeant d'échelle, en allant voir des papiers de famille traitant de questions très locales, comme Debien l'avait déjà fait dans le Haut-Poitou, qu'on peut apercevoir comment le local et le global sont liés, comment des coins du Poitou, de la Bretagne ou de la Basse-Navarre furent en relation directe avec l'Amérique. Dans mon étude, en observant les choses à l'échelle individuelle et familiale, j'ai pu mettre en évidence plusieurs aspects peu remarqués de ces interactions entre colonies et métropole. Par exemple, on dit souvent que les "cadets de Gascogne " émigraient parce que la totalité de l'héritage allait à l'aîné. En réalité, les émigrants pyrénéens, nobles ou paysans, bénéficiaient du soutien financier du groupe familial pour s'installer dans la colonie. En ce sens, mon but est moins d'établir des rapports de cause à effet (le droit d'aînesse absolue est-il la cause de l'émigration pyrénéenne vers SaintDomingue au $18^{\mathrm{e}}$ siècle ?) que de montrer quel est le sens de certains comportements rapportés à certaines règles et coutumes. Par exemple, lorsqu'il émigra à Saint-Domingue en 1764, Jean-Pierre Lamerenx souhaita garder son statut d'héritier de la maison d'UhartJuzon en Basse-Navarre. Je montre que ce choix était à la fois conforme aux coutumes successorales et en conflit avec elles. Le droit d'aînesse était respecté. En revanche, ce choix se faisait dans une logique d'expansion du patrimoine qui était contraire aux coutumes pyrénéennes favorisant le statut quo. De plus, c'était la proximité, plus que l'aînesse en elle-même, qui légitimait les aînés comme héritiers. D'où un conflit au moment de la succession entre Lamerenx et sa sœur cadette qui était restée en Basse- 
Navarre et arguait de sa proximité pour hériter de la «maison ». Je montre ici comment le fait colonial fut générateur de conflits et de situations nouvelles dans des sociétés qui sembleraient n'avoir eu avec l'Amérique que des rapports très lointains. Autre exemple : Jean Mouscardy, cadet de la maison Mouscardy, exploitation agricole de taille moyenne, émigra à Saint-Domingue dans les années 1740. Comme l'explique Anne Zink, un des aspects essentiels des coutumes successorales pyrénéennes était que le cadet ne pouvait pas s'installer à proximité de la maison dont héritait l'aînéee. ${ }^{10}$ Cependant, ayant fait fortune comme planteur de café, Jean Mouscardy investit une partie de ses profits en achetant une ferme à proximité de celle dont avait hérité sa sœur aînée, «dame de la maison Mouscardy ». Jean Mouscardy étant décédé en 1797 sans enfants légitimes, la ferme revint à son frère cadet. Ici encore, le fait colonial donne lieu à une situation inédite : deux « maisons » voisines appartenant aux membres d'une même fratrie.

\section{Le sens d'un titre : Richesse et désastre}

Je souhaite dire un mot surle sens du titre, Wealth and Disaster. Ce qui m'a paru producteur de sens dans le choix de ces deux mots est qu'on a affaire à une fausse opposition. Le titre est signifiant parce qu'il est bancal. Dans un titre comme celui du livre de Donald Winch sur l'économie politique anglaise, Riches and Poverty ${ }^{11}$, on a affaire à une opposition franche: la richesse est opposée à la misère. Lorsqu'on oppose richesse et désastre dans le contexte de la colonisation de Saint-Domingue, on pense bien sûr aux fortunes colossales des colons qui s'évaporèrent suite à ce qui fut de leur point de vue le désastre de la Révolution haïtienne. Mais en déplaçant le point de vue, le désastre peut être au contraire une des conditions de l'accumulation des richesses: le désastre de l'extermination des Tainos, habitants autochtones d'Hispaniola, et le désastre du passage $\mathrm{du}$ milieu. Si on considère l'histoire de la famille Lamerenx sur plusieurs générations, richesse et désastre s'opposent et se conjuguent successivement et parfois même simultanément. Le premier émigrant de la famille, Pierre Lamerenx, calviniste comme le reste de la noblesse béarnaise, quitta le Béarn en 1685 pour s'engager comme enseigne sur un navire de la Compagnie néerlandaise des Indes orientales et mourut à Batavia en 1700. Son émigration fut la conséquence immédiate du désastre de la révocation de l'Édit de Nantes. L'émigration vers Saint-Domingue de son neveu Marc-Antoine en 1729 fut facilitée par les mêmes réseaux qui avaient permis d'exfiltrer une partie des élites béarnaises dans les années 1680. Les enfants et les neveux de Marc-Antoine furent contraints de quitter Saint-Domingue en conséquence du désastre qu'était, de leur point de vue, la révolte de leurs esclaves. Mais c'est ce désastre même qui fut à l'origine de nouvelles fortunes à Cuba. Si l'on s'interroge sur les mécanismes du commerce colonial, on voit toute la complexité des rapports entre richesse et désastre ou, pour reprendre le titre de l'ouvrage classique de Richard Pares, guerre et commerce ${ }^{12}$. Dans un système colonial mercantiliste, l'accumulation du capital se fait par l'établissement de monopoles. Selon le système de l'Exclusif en France ou des Navigation Acts en Angleterre, les colonies sont contraintes de commercer uniquement avec la métropole. C'est cette situation de monopole, conjuguée à l'exploitation du travail servile, qui explique les profits spectaculaires des marchands et des planteurs. Or, si un monopole est relativement facile à faire respecter par les forces de police dans le territoire métropolitain d'un état soumis à un gouvernement donné, il est beaucoup plus difficile à mettre en œuvre dans le commerce international. Un état européen qui décrète une zone de commerce exclusif 
dans une partie des Caraïbes fait face immédiatement à des pirates, à des contrebandiers, et aux forces navales et terrestres des états rivaux, qui sont tout à la fois des concurrents et des ennemis. La mise en œuvre du monopole nécessite l'usage de la force : il faut une marine puissante pour protéger sa zone de commerce exclusif. Il est même rationnel, dans un tel contexte, d'augmenter ses profits en éliminant physiquement ses concurrents : si je capture ou que je coule un vaisseau d'un pays concurrent exportant du sucre ou du café vers l'Allemagne ou la Russie, je pourrai vendre mon café ou mon sucre plus cher dans ces pays. En ce sens, il est nécessaire de faire la guerre pour protéger son monopole, et l'usage de la force est une condition nécessaire à l'accumulation des profits monopolistiques. D'un autre côté, le commerce a besoin de la paix pour prospérer. Les prises de guerre, les blocus, et les opérations des corsaires nuisent considérablement au commerce. Le commerce à longue distance est extrêmement vulnérable aux conflits entre les puissances coloniales. On peut dire à cet égard que la guerre est tout à la fois un facteur nécessaire à l'accumulation du capital et un obstacle majeur à cette accumulation.

C'est là un des points principaux sur lesquels mes analyses rejoignent celles de Paul Cheney, qui montre à quel point le commerce du sucre était vulnérable aux hostilités franco-anglaises. Pendant la guerre d'indépendance américaine, la plantation de La Ferronnays se trouva au bord de la faillite parce que le cours du sucre s'était effondré et que le propriétaire n'avait plus les revenus nécessaires au remboursement des emprunts effectués pour acheter esclaves et machines. Pour ma part, j'étudie le témoignage de Marc-Antoine Lamerenx, qui parle de la guerre de Sept Ans comme d'une «calamité » et résume la situation à deux prix : le cours du café, qui s'était effondré, et le prix de la farine importée de France, multiplié par cinq ou six par rapport au temps de paix. Je calcule que, selon Lamerenx, en temps de guerre une livre de farine valait huit livres de café. Assurément, ces chiffres ne représentent qu'un aspect d'une situation complexe, et l'on sait que dans ces temps de guerre on avait affaire à un important « commerce avec l'ennemi ». Les colons de Saint-Domingue importaient clandestinement de New York et de Philadelphie les farines qu'ils ne pouvaient pas faire venir de Bordeaux ${ }^{13}$. Il reste que mon travail, comme celui de Paul Cheney, contribue à contester une vision un peu trop irénique de l'histoire atlantique telle qu'elle s'est constituée dans les années 80 et 90 . Bernard Bailyn, observant les choses à une très grande altitude, met l'accent sur la stabilité des échanges dans l'espace atlantique au $18^{\mathrm{e}}$ siècle ${ }^{14}$. Il semble voir principalement dans le mercantilisme une théorie économique erronée, remarquable surtout par l'impuissance des gouvernements à arrêter le commerce interlope. Cependant, une toute autre image, faite de discontinuités et de ruptures, apparaît lorsque l'on change d'échelle. Si l'on se situe à l'échelle des individus ou des familles, l'histoire atlantique est celle de catastrophes à répétition. Comme Cheney, je suis d'avis que cette instabilité était une conséquence de l'instabilité structurelle du commerce colonial qui nécessitait simultanément et contradictoirement la paix et la guerre, comme le montrent les travaux classiques d'Eli Heckscher ${ }^{15}$ ou de Richard Pares. L'histoire est toujours fille de son époque. L'histoire atlantique est devenue un paradigme dominant dans les années 90 , époque de discours optimistes surla mondialisation. Si l'on cherche des études historiques prenant le mercantilisme au sérieux, il faut remonter aux années 30 , époque d'intense «jalousie du commerce » (jealousy of trade), pour reprendre une expression de Hume citée par István Hont ${ }^{16}$. 


\section{Capitalisme et esclavage}

18 J'en viens à présent au débat entre l'auteur de Cul de Sac et les auteurs de The Plantation Machine. John Garrigus et Trevor Burnard décrivent l'économie de plantation comme un système efficace, engendrant des profits considérables fondés sur une exploitation implacablement rationnelle de la main d'œuvre servile. Paul Cheney insiste pour sa part sur l'aspect précapitaliste de l'économie de plantation, son caractère paternaliste, la recherche systématique du monopole et de la rente, et aussi l'extrême fragilité du commerce colonial, dont les profits s'évanouissaient en temps de guerre. Je ne prétends pas ici trancher le débat. J'offrirai simplement quelques éléments pour approfondir la réflexion.

19 Allant dans le sens de l'interprétation de Burnard et Garrigus, un détail que j'ai remarqué dans mes recherches, quoique je ne l'analyse pas dans mon livre: les colons de SaintDomingue désignaient souvent leurs exploitations comme des "manufactures ». J'en donnerai un seul exemple, tiré des archives que j'ai consultées. En 1793, la fille de MarcAntoine Lamerenx, refugiée dans la partie espagnole de Saint-Domingue, adressa une demande de secours au capitaine général, Joaquín García y Moreno. Dans sa lettre elle décrit sa plantation comme une " manufacture à café » :

Propriétaire d'une des plus belles manufactures à café, située au quartier de la Guille, paroisse du Dondon, ses nègres il y a deux ans passés se sont révoltés et l'auraient égorgée sans l'heureuse assistance du commandant espagnol près duquel elle s'est réfugiée depuis ce temps. C'est à St. Raphaël qu'elle jouit de cette tranquillité dont sa malheureuse patrie est privée ${ }^{17}$.

On range aujourd'hui le sucre et le café dans la catégorie des produits agricoles. Il est intéressant de constater que, dans l'esprit des colons de Saint-Domingue, les plantations étaient des exploitations industrielles. La feuille de canne et la baie de caféier demandaient en effet des transformations considérables pour obtenir des produits commercialisables. Ces transformations s'effectuaient à l'aide de machines, dans des ateliers soumis à une division du travail.

21 Il existe une abondante littérature sur le caractère industriel de la production du sucre, accompagnée d'un débat sur l'absence ou la présence de progrès technique et sur la façon de mesurer les gains de productivité1 ${ }^{18}$. Un passage de Marx est souvent cité dans ce débat, qui désigne la traite négrière et l'économie de plantation comme l'un des aspects essentiels de la phase primitive d'accumulation du capital :

La découverte des contrées aurifères et argentifères d'Amérique, l'extermination et l'asservissement de la population indigène, son ensevelissement dans les mines, les débuts de la conquête et du sac des Indes orientales, la transformation de l'Afrique en garenne commerciale pour la chasse aux peaux noires, voilà de quoi est faite l'aurore de l'ère de la production capitaliste. Ces processus idylliques sont des moments majeurs de l'accumulation initiale ${ }^{19}$.

Dans l'interprétation marxiste, l'origine du profit capitaliste est dans le surtravail, c'est-àdire dans le travail que l'ouvrier effectue en sus de ce qui est nécessaire pour assurer la production et la reproduction de sa propre force de travail. Selon Marx, « seule la forme sous laquelle ce surtravail est extorqué au producteur immédiat, l'ouvrier, distingue les formations sociales économiques, par exemple la société esclavagiste de celle du travail salariée ${ }^{20} »$. En ce sens, la seule différence entre l'économie industrielle du $19^{\mathrm{e}}$ siècle et l'économie de plantation résiderait dans le passage de l'esclavage au salariat. Selon Marx, 
cependant, le salariat est une forme d'extraction du surtravail incomparablement plus efficace que l'esclavage, car le surtravail est évident dans l'esclavage, alors qu'il est caché dans le salariat :

La forme salaire efface donc toute trace de la division de la journée de travail en travail nécessaire et surtravail, en travail payé et travail non payé. La totalité du travail apparaît comme du travail payé. Dans la corvée féodale, le travail que le corvéable fait pour lui-même et le travail forcé qu'il fait pour le propriétaire se distinguent d'une façon tangible et sensible dans le temps et dans l'espace. Dans le travail des esclaves, même la partie de la journée de travail où l'esclave ne fait que remplacer la valeur de ses propres moyens de subsistance, la partie donc où, en réalité, il travaille pour lui-même, apparaît comme du travail pour son maître. Tout son travail apparaît comme du travail non payé. Dans le travail salarié, au contraire, même le surtravail, ou travail non payé, apparait comme payé. Dans le premier cas, le rapport de propriété masque le fait que l'esclave travaille pour luimême, dans l'autre, le rapport d'argent masque le fait que l'ouvrier salarié travaille gratuitement ${ }^{21}$.

Le surtravail est tellement visible dans l'esclavage que, même lorsque l'esclave travaille pour lui-même (pour produire et reproduire sa propre force de travail), il pense travailler pour son maître. D'après Marx, l'esclave n'a en ce sens aucune raison d'être soigneux dans son travail, et le fait qu'il maltraite ses instruments de travail est une manière de manifester sa dignité humaine vis-à-vis de l'outil inanimé22 . C'est là, remarque Marx, « un des facteurs qui renchérissent la production fondée sur l'esclavage ${ }^{23}$.» En faisant cette remarque, Marx est dans la lignée de nombreux économistes du $18^{\mathrm{e}}$ siècle, Adam Smith en tête, qui critiquaient l'esclavage comme une forme de travail inefficace et non rentable. D'autres économistes, dont Jean-Baptiste Say, soutenaient au contraire que l'économie de plantation avait été extrêmement rentable, et que l'exploitation du travail servile entrait pour une bonne part dans les profits des planteurs ${ }^{24}$. Selon Adam Smith, c'est parce que le commerce colonial était fondé sur le monopole qu'il engendrait des profits spectaculaires, et c'est l'existence de ces profits monopolistiques qui permettait aux planteurs d'avoir recours au type de travail inefficace qu'était l'esclavage ${ }^{25}$. Si le monopole était aboli, les planteurs seraient obligés de recourir au travail salarié. D’après Jean-Baptiste Say, les profits des planteurs venaient en partie du monopole et en partie de l'exploitation du travail servile ${ }^{26}$. Il est bien sûr extrêmement difficile de trancher cette question empiriquement. On a de nombreux témoignages de retours sur investissement de l'ordre de 10 à $15 \%$ par $a^{27}$, mais il est presque impossible de déterminer quelle part du profit est attribuable au monopole. Et on sait par ailleurs, comme le souligne Cheney, que, en temps de guerre, les retours sur investissement étaient négatifs. Je souscrirais pour ma part assez volontiers au jugement d'Eric Williams : «Des richesses considérables furent produites par une économie instable fondée sur la monoculture, qui avait tous les vices de la féodalité et du capitalisme et aucune de leurs vertus ${ }^{28}$.»

\section{Oncles d'Amérique}

Un mot enfin sur la figure de l'oncle d'Amérique, par laquelle j'ai choisi d'ouvrir et de fermer mon livre. Dans la préface, je cite la pièce d'Eugène Scribe, L'Oncle d'Amérique, créée à Paris en 1826, peu de temps après la reconnaissance de l'indépendance d'Haïti par Charles $\mathrm{X}$ et l'annonce d'une indemnité versée par le gouvernement haïtien au bénéfice des anciens colons. Je montre que la pièce fait allusion à ce contexte, et il apparaît que la figure de l'oncle d'Amérique, associée aujourd'hui dans l'imagination populaire aux 
fortunes rapides faites aux États-Unis, est historiquement liées aux fortunes faites (et défaites) à Saint-Domingue. Dans les archives que j'ai consultées, j'ai trouvé un témoignage de l'impact des fortunes faites à Saint-Domingue sur l'imagination populaire dans des zones rurales qui sembleraient à première vue avoir peu de rapports avec le grand large (un seul témoignage mais, comme le souligne Carlo Ginzburg, en microhistoire, la qualité de l'information est plus importante que sa quantitée ${ }^{29}$ ). Lorsque JeanPierre Lamerenx, propriétaire d'une caféière au Dondon, revint en Basse-Navarre en 1787 pour s'occuper de la succession de ses parents, il était accompagné de trois de ses enfants, dont une fille, Marguerite, qu'il confia à sa sœur et à son beau-frère pour qu'ils l'éduquent en France. Vingt ans plus tard, en 1808, Marguerite voulut se marier, mais il fut impossible d'obtenir son acte de naissance, vu l'absence de relations diplomatiques avec la nouvelle république d'Haïti. Un juge de paix dut conduire une enquête de notoriété pour prouver que la jeune femme était bien née à Saint-Domingue. Plusieurs témoins furent interrogés à propos du moment où le père et la fille firent leur apparition en BasseNavarre, en grand équipage, et en multipliant les signes extérieurs de richesse (y compris, très probablement, des esclaves à leur suite). "L'arrivée de M. Lamerenx, » déclare un témoin, » fit beaucoup d'éclat et attira nombre de curieux pour le voir. » Vingt ans après les faits, le souvenir du luxe ostentatoire d'un "Américain » était encore bien présent dans les imaginations. Il est clair par ailleurs que, dès cette époque, l'image de l'oncle d'Amérique était très ambiguë, et que les anciens colons inspiraient un mélange contradictoire de respect et de mépris, en tant qu'anciens propriétaires d'esclaves qui étaient peut-être riches ou peut-être pauvres, et venaient d'un monde désormais disparu. En m'attaquant à la conclusion de mon livre, j'ai eu envie de citer le film d'Alain Resnais, Mon oncle d'Amérique, pour montrer que, vers la fin du $20^{\mathrm{e}}$ siècle, l'idée de devenir riche en Amérique était perçue comme un pur fantasme et une rêverie enfantine, et qu'il y avait aussi dans cette représentation un côté sombre (le film de Resnais finit sur des images du Bronx à la fin des années 70, paysage sinistre de ville en ruines). Il est frappant de constater que les premières représentations de l'Amérique comme contrée dystopique remontent au $18^{\mathrm{e}}$ siècle, et en particulier aux descriptions de Saint-Domingue par Stanislas de Wimpffen (pays sinistre où la richesse n'est qu'apparente, et où tout est souillé par la présence de l'esclavage ${ }^{30}$. Je me suis demandé s'il n'existait pas un lien souterrain entre le scénario du film de Resnais et le souvenir de Saint-Domingue. Après avoir cherché un peu j'en ai trouvé un, qui ne peut pas être le fait du hasard. Dans le film de Resnais, il est question du "roi de l'or ", héros d'un livre illustré pour enfants des années $1920^{31}$. Ce roi de l'or est un jeune homme d'origine française qui possède une fabrique de lingots d'or aux États-Unis et s'engage dans l'armée française pour combattre les Allemands en 1914. Ici, l'oncle d'Amérique se présente sous forme d'une rêverie enfantine associée aux États-Unis. Il se trouve que l'auteur du Roi de l'or, Alice Pujo (1869-1953), était la petite-fille d'un certain Alexis-Solange Pujo, originaire des HautesPyrénées, et mentionné dans l'Indemnité de Saint-Domingue ${ }^{32}$ comme propriétaire d'une plantation de café au Dondon qui jouxtait les plantations de mes personnages principaux, Marc-Antoine Lamerenx et Jean Mouscardy. ${ }^{33}$ J'ignore si Alice Pujo était consciente de ce passé colonial, mais il y a là, me semble-t-il, plus qu'une simple coïncidence. 


\section{NOTES}

1. Pierre FORCE, Wealth and Disaster. Atlantic Migrations from a Pyrenean Town in the Eighteenth and Nineteenth Centuries, Baltimore, Johns Hopkins University Press, 2016.

2. Paul CHEney, Cul de Sac. Patrimony, Capitalism, and Slavery in French Saint-Domingue, Chicago, University of Chicago Press, 2017.

3. Trevor BURNARD et John GARRIGUS, The Plantation Machine. Atlantic Capitalism in French SaintDomingue and British Jamaica, Philadelphie, University of Pennsylvania Press, 2016.

4. Voir notamment Jacques DE CAUNA, L'Eldorado des Aquitains. Gascons, Basques et Béarnais aux îles d'Amérique (XVII -XVIII siècles), Biarritz, Atlantica, 1998.

5. Voir Jean-Claude PASSERON et Jacques REVEL, Penser par cas. Raisonner à partir de singularités, Paris, EHESS, 2005.

6. Pierre Bourdieu, Le Bal des célibataires. Crise de la société paysanne en Béarn, Paris, Seuil, 2002

7. Henri lefebvre, Les Communautés paysannes pyrénéennes, Orthez, Société Ramond / Cercle historique de l'Arribère, 2014 ; La Vallée de Campan. Étude de sociologie rurale, Paris, PUF, 1963.

8. Fréderic MAURO, « Petit monde antillais. À Saint-Domingue au XVIII ${ }^{\mathrm{e}}$ siècle », Annales 3-4 (1948), p. 532.

9. Fernand BRAUDEL, « Antilles et Amérique », Annales 3-4 (1948), op. cit., p. 537.

10. Anne ZINK, L'Héritier de la maison. Géographie coutumière du Sud-Ouest de la France sous l'ancien régime, Paris, EHESS, 1993, p. 262.

11. Donald WINCH, Riches and Poverty. An Intellectual History of Political Economy in Britain, 1750-1834, Cambridge, Cambridge University Press, 1996.

12. Richard PARES, War and Trade in the West Indies, 1739-1763, Oxford, Clarendon Press, 1936.

13. Thomas M. TRUXES, Defying Empire: Trading with the Enemy in Colonial New York, New Haven, Yale University Press, 2008.

14. Bernard BAILyN, Atlantic History. Concepts and Contours. Cambridge, Harvard University Press, 2005, p. 84.

15. Eli F. HECKSCHER, Mercantilism, Londres, Allen \& Unwin, 1935. Une exception récente est Mercantilism Reimagined. Political Economy in Early Modern Britain and Its Empire, sous la direction de Philip J. Stern et Carl Wennerlind, Oxford, Oxford University Press, 2013.

16. István HONT, Jealousy of Trade. International Competition and the Nation-State in Historical Perspective, Cambridge, Cambridge University Press, 2005.

17. Marguerite-Françoise Lamerenx, veuve Saint-Germain, à Joaquín García y Moreno, capitaine général de Santo Domingo, 18 septembre 1793, Archivo General de Sevilla, Secretaría del Despacho de Guerra, Santo Domingo, SGU LEG 7158.

18. Stuart B. SCHWARTz (sous la direction de), Tropical Babylons. Sugar and the Making of the Atlantic World, Chapel Hill, University of North Carolina Press, 2004 ; Sidney MINTZ, Sweetness and Power. The Place of Sugar in Modern History, New York, Penguin Books, 1985 ; Eric WILLIAMS, Capitalism and Slavery, Chapel Hill, University of North Carolina Press, 1944.

19. Karl MARX, Le Capital, traduction sous la direction de Jean-Pierre Lefebvre, Paris, PUF, 1993, Livre I, Chap. XXIV, p. 893.

20. K. MARX, Le Capital, op. cit., Livre I, Chap. VII, p. 243.

21. K. MARX, Le Capital, op. cit., Livre I, Chap. XVII, p. 604-605.

22. K. MARX, Le Capital, op. cit., Livre I, Chap. V, p. 220. 
23. Ibid.

24. Sur ce débat dans le contexte français, voir Caroline oudin-BASTIDE et Philippe STEINER, Calcul et morale. Coûts de l'esclavage et valeur de l'émancipation (18 $18^{e}-19^{e}$ siècle), Paris, Albin Michel, 2015.

25. Adam SMITH, Richesse des nations, III.ii.10.

26. Jean-Baptiste SAY, Traité d'économie politique, Paris, Deterville, 1803, livre I, chapitre 28.

27. Pierre FORCE, Wealth and Disaster, op. cit., p. 55.

28. «Tremendous wealth was produced from an unstable economy based on a single crop, which combined the vices of feudalism and capitalism with the virtues of neither. ». Eric Williams, The Negro in the Caribbean. Bronze Booklet No. 8, Washington, D.C., The Associates in Negro Folk Education, 1942, p. 13.

29. Carlo GINZBURG, Mythes, emblèmes, traces. Morphologie et histoire. Paris, Flammarion, 1989.

30. Alexandre STANISLAS DE WIMPFFen, Voyage à Saint-Domingue pendant les années 1788, 1789, et 1790, vol. 2, Paris, Cocheris, 1797.

31. Alice PUjo, Le Roi de l'or, Paris, Bonne presse, 1920.

32. État détaillé des liquidations opérées à l'époque du 1er janvier 1828-1832 et les six premiers mois de 1833, par la Commission chargée de répartir l'indemnité attribuée aux anciens colons de Saint-Domingue, en exécution de la loi du 30 avril 1826 et conformément aux dispositions de l'ordonnance du 9 mai suivant, 6 vols, Paris, Imprimerie royale, 1828-1833, vol. 3, p. 88-89.

33. P. FORCE, Wealth and Disaster, op. cit., p. 156.

\section{RÉSUMÉS}

L'auteur de Wealth and Disaster rend compte des circonstances de la conception de son ouvrage et revient sur ses conclusions ainsi que sa méthode. L'origine du livre est une trouvaille archivistique. Plutôt que de confirmer ou infirmer une hypothèse de grande envergure, il s'agissait d'examiner, selon la méthode micro-historique, quelques « cas ", c'est-à-dire des faits qui semblent contrevenir aux normes d'un lieu ou d'une époque (la Basse-Navarre au $18^{\mathrm{e}}$ siècle). L'auteur pose la question de savoir pourquoi deux cadets, que les coutumes successorales pyrénéennes destinaient à la pauvreté, firent fortune à Saint-Domingue, et pourquoi un aîné prit la décision d'émigrer, alors que l'émigration était normalement le fait des cadets. Une des conclusions principales est de montrer l'impact du fait colonial sur les coutumes successorales de zones rurales qui sembleraient à première vue peu sensibles aux soubresauts $\mathrm{du}$ monde atlantique. L'article souligne les convergences de cet ouvrage avec celui de Paul Cheney, Cul-deSac, en particulier sur la vulnérabilité des plantations de sucre et de café au blocus et à la guerre, et il offre quelques réflexions sur les rapports entre capitalisme et esclavage tels qu'ils étaient compris aux $18^{\mathrm{e}}$ et $19^{\mathrm{e}}$ siècles.

The author of Wealth and Disaster provides an account of how his book came about, and he reflects on his conclusions as well as his method. The book arose from an archival find. Rather than seeking to prove or disprove a large-scale hypothesis, the book follows a micro-historical method in examining a few "cases", i.e. facts that seem to go against the norms of a particular time and place (Lower Navarre in the $18^{\text {th }}$ century). The author asks why two younger sons made their fortunes in Saint-Domingue even though they were destined to poverty in accordance to Pyrenean inheritance customs, and why a first-born decided to emigrate even though emigration 
was normally restricted to younger sons. One of the main conclusions is to show the impact of the colonial reality on the inheritance customs of rural zones that would initially seem immune to the upheavals of the Atlantic world. Emphasis is given to the convergence between this study and Paul Cheney's Cul-de-Sac, especially regarding the vulnerability of sugar and coffee plantations to blockade and war. Finally, some reflections are offered on the relationship between capitalism and slavery as it was understood in the $18^{\text {th }}$ and $19^{\text {th }}$ centuries.

INDEX

Keywords : migration, Pyrenees, Saint-Domingue, inheritance customs, capitalism, slavery Mots-clés : émigration, Pyrénées, Saint-Domingue, coutumes successorales, capitalisme, esclavage

\section{AUTEUR}

\section{PIERRE FORCE}

Columbia University 